\title{
Chronological expression of Ciliated Bronchial Epithelium 1 during pulmonary development
}

\author{
H.M. Haitchi*, H. Yoshisue*, A. Ribbene*,\#, S.J. Wilson*, J.W. Holloway*, , \\ F. Bucchieri*,\#, N.A. Hanley", D.I. Wilson", G. Zummo" ${ }^{\#}$, S.T. Holgate* and D.E. Davies*
}

ABSTRACT: Ciliated Bronchial Epithelium (CBE) 1 is a novel gene, which is expressed in ciliated cells. As cilia are important during embryogenesis, the present authors characterised the murine homologue of CBE1 (Cbe1) and compared its temporal expression during murine and human lung development.

Cbe1 cDNA was cloned and characterised using sequencing, standard PCR and Western blotting. Mouse and human embryonic/fetal lungs (HELs) were harvested for mRNA analysis and protein localisation in vivo and in vitro using RT-PCR and immunohistochemistry.

The Cbe1 amino acid sequence was $>75 \%$ identical with $\mathrm{CBE} 1$ and its alternative splicing and tissue distribution were highly conserved. Pulmonary expression of Cbe1 mRNA was increased at embryonic day (E)16, 1 day later than Foxj1, which is consistent with a role in ciliogenesis. In HELs, CBE1 mRNA was detectable at 8-9 weeks post-conception and increased in explant culture. CBE1 protein expression was weak at 10 weeks post-conception but strong at 12.3 weeks post-conception, in parallel with cilia formation. Additionally, Cbe1 mRNA was expressed at E11 (4-5 weeks post-conception in HELs) in the absence of Foxj1, implying a distinct role in early development.

Chronological regulation of $\mathrm{CBE1} / \mathrm{Cbe1}$ expression during pulmonary differentiation suggests involvement in ciliogenesis, with an additional role during early lung development.

KEYWORDS: Ciliated bronchial epithelium 1, ciliogenesis, embryonic/fetal lung development, epithelium, forkhead box factor $\mathrm{J1}$

ilia are finger-like appendages that are microtubule (MT)-based organelles. They are classified according to their MT components as 9+2 (motile) and 9+0 (primary) cilia. The airway is the archetypal tissue containing motile cilia. The ciliated cells in the tracheal and bronchial epithelium of the lower airways play a pivotal role in propelling mucus secretions towards the pharynx [1, 2]. Although the molecular mechanisms of epithelial ciliogenesis have not been fully investigated, the transcription factor forkhead box factor (FOX) J1 (hepatocyte nuclear factor-3/forkhead homologue 4) is closely involved in ciliogenesis. Targeted disruption of the Foxj1 gene in mice results in an absence of airway cilia and situs inversus [3, 4], suggesting that Foxj1 is important not only for the differentiation of airway epithelium but also for the normal positioning of internal organs. However, forced over expression of Foxj1 in undifferentiated airway epithelial does not induce formation of ciliated cells [5], implying that Foxj1 alone is not sufficient for the development of cilia and that ciliogenesis requires other different transcription factors, which have yet to be characterised.

Recently, a novel gene was characterised, Ciliated Bronchial Epithelium (CBE) 1, which was initially identified as a differentially represented gene in cDNA libraries derived from asthmatic and normal bronchial biopsies [6]. Although its predicted amino acid sequence has no similarity to known proteins, expression of CBE1 is strongly associated with ciliated epithelial cells both in bronchial and nasal tissues. Importantly, immunostaining was observed intracellularly but not within the ciliary structure, suggesting that CBE1 does not constitute a component of cilia.

This article has supplementary material accessible from www.erj.ersjournals.com

AFFILIATIONS

*Division of Infection, Inflammation and Repair, School of Medicine University of Southampton, Southampton General Hospital, and "Centre for Human Development, Stem Cells and Regeneration, Human Genetics Division, School of Medicine, University of Southampton, Southampton General Hospital, Southampton, UK.

"Human Anatomy Section, University of Palermo, Palermo, Italy.

CORRESPONDENCE

D.E. Davies

Division of Infection, Inflammation and Repair, School of Medicine, University of Southampton

Southampton General Hospital,

Tremona Road

Southampton

S016 6YD

UK

Fax: 442380701771

E-mail: donnad@soton.ac.uk

Received:

October 172008

Accepted after revision:

December 182008

\section{SUPPORT STATEMENT}

The authors received support from the Asthma, Allergy and Inflammation Research (AAIR), Hope Charities and Roger Brooke Charitable Trust.

STATEMENT OF INTEREST None declared. 
Expression studies showed that CBE1 is localised to the nuclear or perinuclear regions of cells, implying that CBE1 might be a nucleocytoplasmic shuttling protein, although no clear function for CBE1 has been described as yet. Although strong induction of the CBE1 mRNA during in vitro mucociliary differentiation of primary bronchial epithelial cells has been shown, its expression during lung development has not been investigated. The present authors have characterised the mouse ortholog of CBE1 (Cbe1), analysed chronological expression of Cbe1 mRNA during pulmonary differentiation in vivo and compared this with CBE1 mRNA and protein expression in human embryonic/fetal lung (HEL) explants cultured in vitro.

\section{MATERIALS AND METHODS}

\section{Cloning and characterisation of Cbe1 cDNA}

cDNA from adult mouse lung was amplified using primers specific for Cbe1 and the products cloned and sequenced. cDNAs encoding open reading frame (ORF)1 and ORF2 were cloned into pcDNA3.1 (Invitrogen, Carlsbad, CA, USA) and transfected into HEK293 cells. Isolation of cellular extracts, SDS-PAGE and Western blotting were performed as described previously [6]. Detailed protocols are provided in the online supplementary data.

\section{Isolation of mouse lungs}

Mouse lungs were harvested at embryonic day (E)11-19, postnatal day 1 and 8 , and from adult mice (AM). Dissected lungs were immediately homogenised in Trizol reagent (Invitrogen) for RNA isolation (see below).

\section{Isolation of human fetal lungs and ex vivo differentiation}

Human fetal lung tissues were collected from females undergoing first trimester termination of pregnancy with informed written consent and ethical approval. Isolated tissues were staged and processed as described previously [7], or cultured in vitro at an Matrigel air-liquid interface (ALI) using Ultraculture serum free medium (Cambrex, Verviers, Belgium) for $\leqslant 18$ days.

\section{Immunohistochemistry}

Human fetal lungs were processed into glycol methacrylate resin and $2-\mu \mathrm{m}$ sections were cut and subjected to immunohistochemical analysis with immunoperoxidase detection using diaminobenzidine or 3-amino-9-ethylcarbazole as chromagens, as previously described [8]. Affinity purified rabbit polyclonal antibody generated against CBE1 [6] was used at $2 \mu \mathrm{g} \cdot \mathrm{mL}^{-1}$.

\section{RNA extraction and RT-PCR}

RNA samples were isolated using Trizol reagent according to the manufacturer's instructions and treated with RNase-free DNase I (Ambion, Huntingdon, UK) to remove any contaminating genomic DNA. cDNA was synthesised as previously described [6]. For semi-quantitative PCR and nested PCR, cDNA was amplified using specific primers as described in the online supplementary data. PCR products were separated in $1.5 \%$ agarose gel and visualised with ethidium bromide or Vistra Green (Amersham Biosciences, Amersham, UK). RTquantitative PCR (RT-qPCR) was performed using an IcyclerIQ system (Bio-Rad, Hemel Hempstead, UK) [9]. Relative expression levels were calculated using the $\Delta \Delta \mathrm{Ct}$ (threshold cycle) method. Specific primers, probes and experimental conditions are provided in the online supplementary data. The results were expressed relative to either Glyceraldehyde-3-phosphate dehydrogenase (Gapdh) or beta-actin (ACTB) mRNA levels, which were used as housekeeping genes.

\section{Statistical analyses}

Statistical analysis was undertaken using the Mann-Whitney test. A p-value $<0.05$ was considered as significant.

\section{RESULTS}

\section{Characterisation of $\mathrm{Cbe} 1$ and its tissue distribution}

To compare the expression profile of CBE1 mRNA with that of its rodent counterpart, the present authors cloned and characterised the murine ortholog of CBE1 (Cbe1). A basic local alignment search tool (BLAST) search using CBE1 as a query easily identified a highly homologous mouse mRNA (accession number AK003742 in the GenBank database), which has not been fully annotated. Specific primers were designed based on the sequence AK003742, and the putative full-length cDNA of Cbe1 was amplified by PCR, followed by sequencing analyses. Figure 1a shows the resulting consensus sequence of the cDNA of Cbe1, within which a small ORF consisting of 126 amino acids was found. The first methionine codon of this ORF is preceded by an in-frame stop codon $42 \mathrm{bp}$ upstream, and is flanked by a Kozak's consensus sequence (A/GXXatgG) [10], suggesting that it is the genuine translation initiation site. Given this, together with a putative polyadenylation signal (AATAAA) in the 3-untranslated region (fig. 1a), this is a fulllength cDNA. Two out of twelve independent clones that were sequence analysed showed a 5-bp insertion at one of the splicing sites, resulting in a frame shift. In turn, this generated another ORF (ORF2) consisting of 162 amino acids with a different carboxyl terminus. It is interesting that the way these splicing variants are generated is completely conserved between mice and human [6]. BLAST search analyses has also identified another splicing variant (accession number XM355478) of Cbe1 harbouring a longer amino-terminus, like $C B E 1$. Specific primers were designed to detect this variant by RT-PCR, clarifying that the longer form is not expressed in lung but is expressed in testis. The longer form also has two splicing variants with different carboxyl termini, due to the 5bp insertion (fig. 1b).

The predicted amino acid sequence of ORF1 of Cbe1 is $75.4 \%$ identical to that of CBE1, whereas $78.4 \%$ identity is found for ORF2 (fig. 1c). Although both ORFs have no obvious similarity to known proteins, the two arginine residues that could serve as one of the nuclear localisation signals in CBE1 [6] are conserved.

The present authors also undertook semi-quantitative RT-PCR to evaluate the tissue distribution of Cbe1 mRNA in adult tissues. This showed that the long form was testis-specific, and that the short form was abundantly expressed in lung and testis (fig. 1b), with relatively lower expression in brain and thymus and no detectable expression in other analysed tissues (heart, liver, spleen or kidney; fig. 2a), This latter finding is in contrast with CBE1 mRNA which is also expressed in heart and kidney [7], although Cbe1 mRNA could be detected in embryonic heart using RT-qPCR (data not shown). These data suggest that expression of Cbe1 mRNA is highly tissue-specific and that its pattern is partially consistent with that of $C B E 1$. 
a)

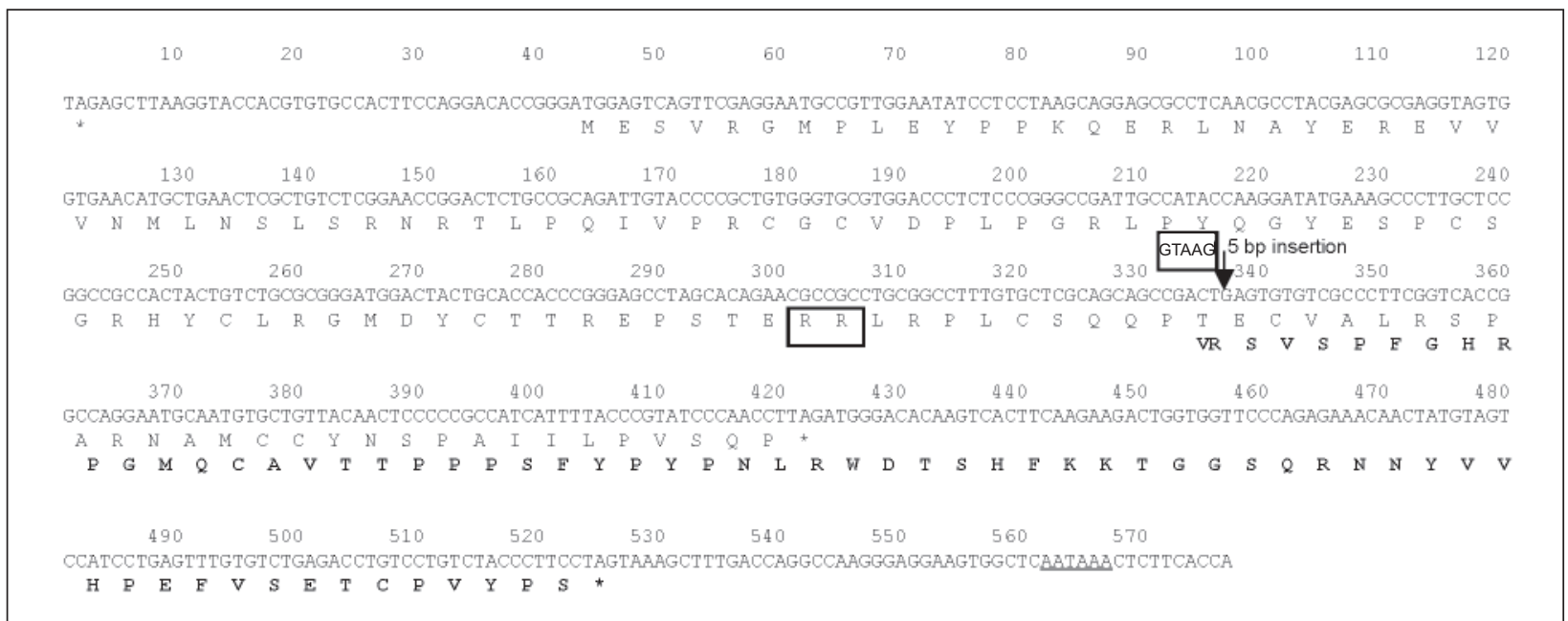

b)
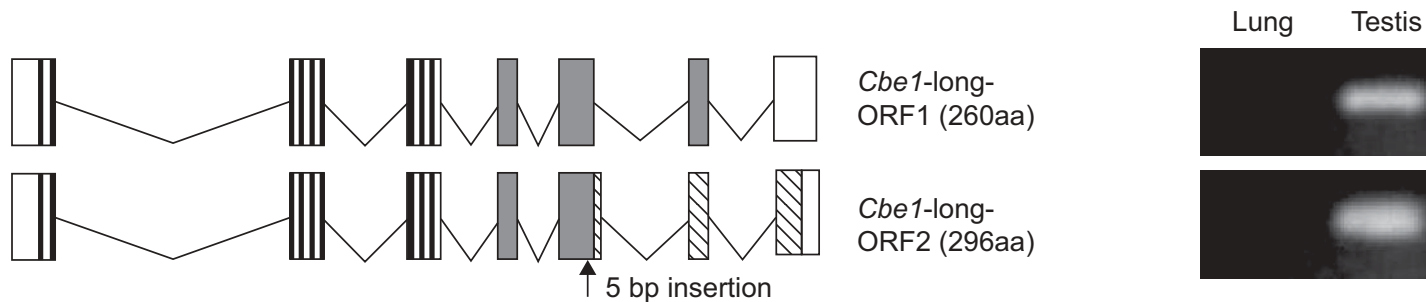

(GTAAG)

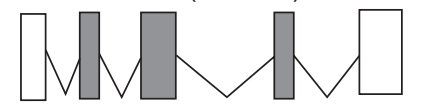

Cbe1-ORF1

(126aa)

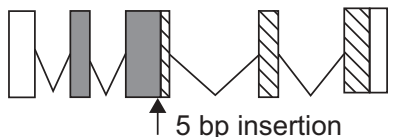

Cbe1-ORF2

(162aa)

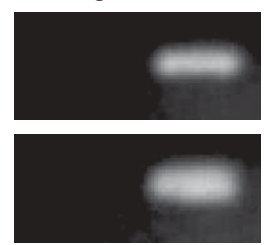

(GTAAG)

c)

\begin{tabular}{|c|c|c|c|c|}
\hline human & CBE1 & ORF1 & 1: METAVRGMP LECP PRPERLNAY EREVMVNMLNS LSRNQQLPRITPRCGCVDP LPGRLPFH & 60 \\
\hline mouse & CBB1 & ORF1 & 1: MESV-RGMP LEYP PKQERLNAY BREVVVNMLNSLSRNRTLPQIVPRCGCVDPLPGRLPYQ 5 & 59 \\
\hline $\begin{array}{l}\text { human } \\
\text { mouse }\end{array}$ & $\begin{array}{l}\text { CBE1 } \\
\text { CBE1 }\end{array}$ & $\begin{array}{l}\text { ORF1 } \\
\text { ORF1 }\end{array}$ & 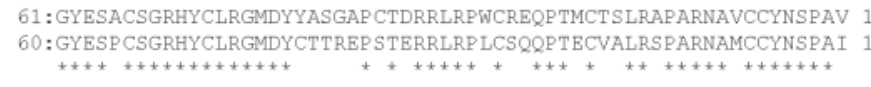 & $\begin{array}{l}120 \\
119\end{array}$ \\
\hline $\begin{array}{l}\text { human } \\
\text { mouse }\end{array}$ & $\begin{array}{l}\text { CBB1 } \\
\text { CBE1 }\end{array}$ & $\begin{array}{l}\text { ORF1 } \\
\text { ORF1 }\end{array}$ & $\begin{aligned} 121 & \text { ILPISEP } \\
120 & \text { ILPVSQP } \\
& * * * * *\end{aligned}$ & $\begin{array}{l}127 \\
126\end{array}$ \\
\hline $\begin{array}{l}\text { human } \\
\text { mouse }\end{array}$ & $\begin{array}{l}\text { CBE1 } \\
\text { CBE1 }\end{array}$ & $\begin{array}{l}\text { ORF2 } \\
\text { ORF2 }\end{array}$ & 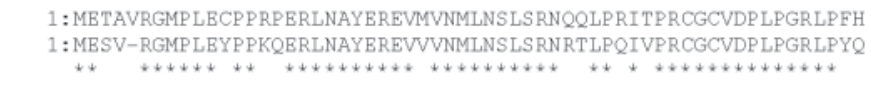 & $\begin{array}{l}60 \\
59\end{array}$ \\
\hline $\begin{array}{l}\text { human } \\
\text { mouse }\end{array}$ & $\begin{array}{l}\text { CBE1 } \\
\text { CBB1 }\end{array}$ & $\begin{array}{l}\text { ORF2 } \\
\text { ORF2 }\end{array}$ & 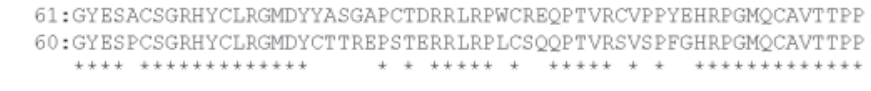 & $\begin{array}{l}120 \\
119\end{array}$ \\
\hline $\begin{array}{l}\text { human } \\
\text { mouse }\end{array}$ & $\begin{array}{l}\text { CBE1 } \\
\text { CBE1 }\end{array}$ & $\begin{array}{l}\text { ORE2 } \\
\text { ORF2 }\end{array}$ & 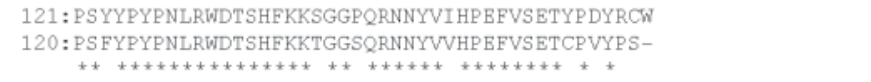 & $\begin{array}{l}164 \\
162\end{array}$ \\
\hline
\end{tabular}

FIGURE 1. Characterisation of the Cbe1 cDNA. a) Nucleotide and deduced amino acid sequences of the lung-expressed full-length Cbe1 cDNA. There is a 5 bpinsertion in one of the splicing sites within the cDNA, leading to an alternative open reading frame (ORF) with a longer and different carboxyl terminus (ORF2; GTAAG). Two arginine residues which were shown to be responsible for its nuclear localisation for CBE1 are shown. GenBank accession number: DQ873295 (ORF1), DQ873296 (ORF2). b) Schematic view of the intron/exon organisation, comparison of the amino acid chain length of splicing variants of Cbe1 mRNA and analysis of their expression in lung and testis. The open boxes represent untranslated regions but the $5^{\prime}$-untranslated sequences of the long and short forms are different to each other due to use of an alternate promoter. Semi-quantitative RT-PCR was carried out using variant-specific primers. PCR cycles were 35 for each Cbe1 mRNA variants. c) Comparison of the amino acid sequences of Cbe1 and CBE1. *: identical residues. 


\section{Regulation of Cbe1 mRNA expression during mouse embryogenesis}

The amino acid sequences in the two synthetic peptide sequences, which were used previously to generate antiCBE1 antibodies, were not completely conserved between CBE1 and Cbe1 [6], with 11 out of 14 amino acids being identical in one peptide and 10 out of 14 identical in the other peptide. As a result, recombinant ORF1 and ORF2 of Cbe1 expressed in HEK293 cells showed much lower reactivity to anti-CBE1 antibodies compared with recombinant CBE1 when
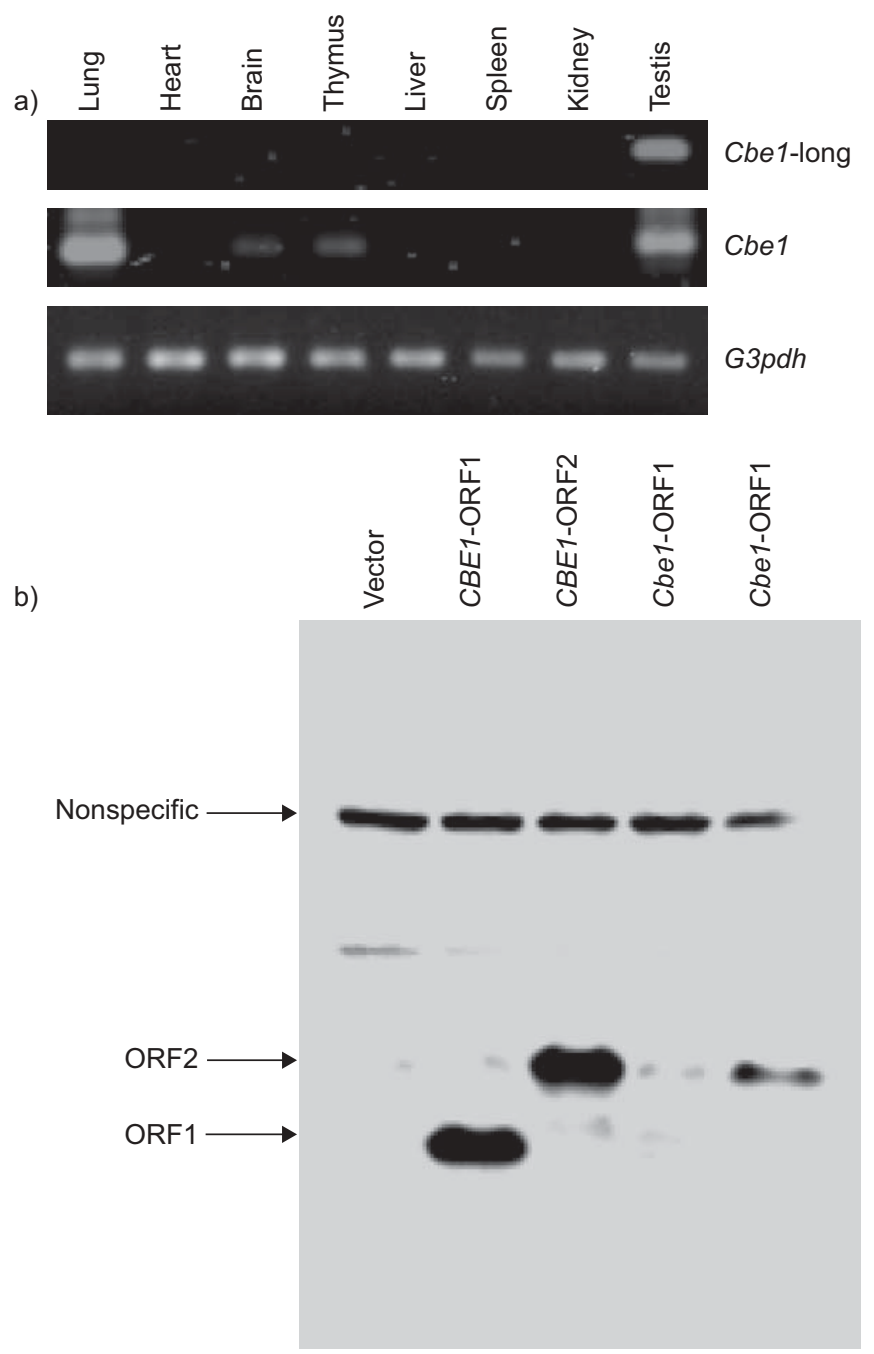

FIGURE 2. a) Tissue distribution of Cbe $1 \mathrm{mRNA}$ analysed by semi-quantitative RT-PCR. Total RNA samples were isolated from indicated different organs of adult mouse, followed by CDNA synthesis and PCR using primers detecting long or short forms (common to open reading frame (ORF)1 and ORF2) of Cbe1. PCR cycles were 35 for Cbe 1 cDNA and 25 for G3pdh cDNA. RT-PCR using variant (ORF1, ORF2)-specific primers resulted in a similar pattern of distribution (data not shown). b) Reactivity of anti-CBE1 antibodies against recombinant CBE1 and Cbe1 proteins. Expression plasmids encoding full-length ORF1 or ORF2 of CBE1 or Cbe1 CDNAs, or vector alone (pcDNA3.1) were transiently introduced into HEK293 cells by lipofection. After $48 \mathrm{~h}$, cellular extracts were separated in 15\% SDS-PAGE, followed by immunoblotting using anti-CBE1 antiserum. Bands of ORF1 and ORF2 are indicated by the arrows. analysed by Western blotting (fig. 2b). Therefore, the present authors chose to use RT-qPCR analyses to investigate the chronological expression of Cbe1 mRNA and other ciliogenesis-related genes during embryogenesis.

Figure 3a shows that transcription of Foxj1 mRNA, which is closely involved in ciliogenesis, was switched on at E15 showing a 15.4 \pm 3.7 -fold induction compared with the basal level at E14. Foxj1 mRNA chronologically increased thereafter, up to $342 \pm 71$-fold in AM. Expression of Cbe1 mRNA increased $15.4 \pm 7.4$-fold at E16 compared with the basal level at E14, which was later than Foxj1, and increased $447 \pm 99$-fold in AM (fig. 3b). In contrast, the expression profile of Foxa1 and Foxa2 mRNAs, forkhead transcription factors closely involved in the differentiation of bronchial epithelium [11, 12], showed little change (Foxa1; fig. 3c) or increased only three to five-fold (Foxa2; fig. 3d) from E11 to adult. This is consistent with a previous report which stated that these transcription factors are expressed from E10.5 [13]. Tektin-1 (Tetk1), a gene encoding proteins which form filamentous polymers in the walls of ciliary and flagellar microtubules [14], showed a $4.5 \pm 1.1$-fold increase in mRNA expression from E15 to E16, which was much less than for Cbe1 or Foxj1 mRNA (fig. 3e). Although the expression profile and similar kinetics of Cbe1 and Foxj1 mRNA during the late pseudoglandular stage of lung development (fig. 3f) are consistent with a role for Cbe1 in ciliogenesis, significantly greater expression of Cbe1 mRNA was observed at E11 compared with E12-E14; however, this was not observed for Foxj1 (fig. 3a and b), suggesting that Cbe1 has a distinct function in early lung development.

\section{Expression of CBE1 in HEL}

The expression of CBE1 mRNA in HEL was analysed using semiquantitative RT-PCR, which showed a low but detectable amount of $C B E 1 \mathrm{mRNA}$ at 10 weeks post-conception. In contrast, FOXJ1 mRNA was consistently observed from 7-10 weeks postconception whereas TEKT1 mRNA was not expressed at 10 weeks post-conception (fig. 4a). In order to detect low copy numbers of CBE1 and TEKT1 mRNA, the present authors performed nested PCR which revealed that CBE1 mRNA was already present at 8-9 weeks post-conception, whereas TEKT1 mRNA was not detectable even with this highly sensitive method (fig. 4b). These results suggest that from 7 weeks post-conception, expression of CBE1 precedes that of TEKT1, but follows that of FOXJ1 mRNA in developing human lung.

The expression of CBE1 protein by immunohistochemistry was also examined using an anti-CBE1 polyclonal antibody. As differentiation of ciliated epithelium in the human airways occurs between 11-16 weeks post-conception in the mid-late pseudoglandular stage $[15,16]$, the present authors used fetal lung tissues obtained at 10 and 12.3 weeks post-conception. Immunoreactivity of the CBE1 protein was hardly detectable in 10 weeks post-conception fetal airway tissue where no cilia were observed (fig. 5a and b). However, expression of CBE1 protein was strong in airway epithelium of lungs at 12.3 weeks post-conception when cilia were clearly visible (fig. $5 c$ and d), and consistent with a correlation of CBE1 expression and ciliogenesis. Positive signals were observed not only in columnar epithelial cells but also in basal epithelial cells of fetal lung (fig. 5c and d), in contrast to adult human bronchi (fig. 5e and f). 

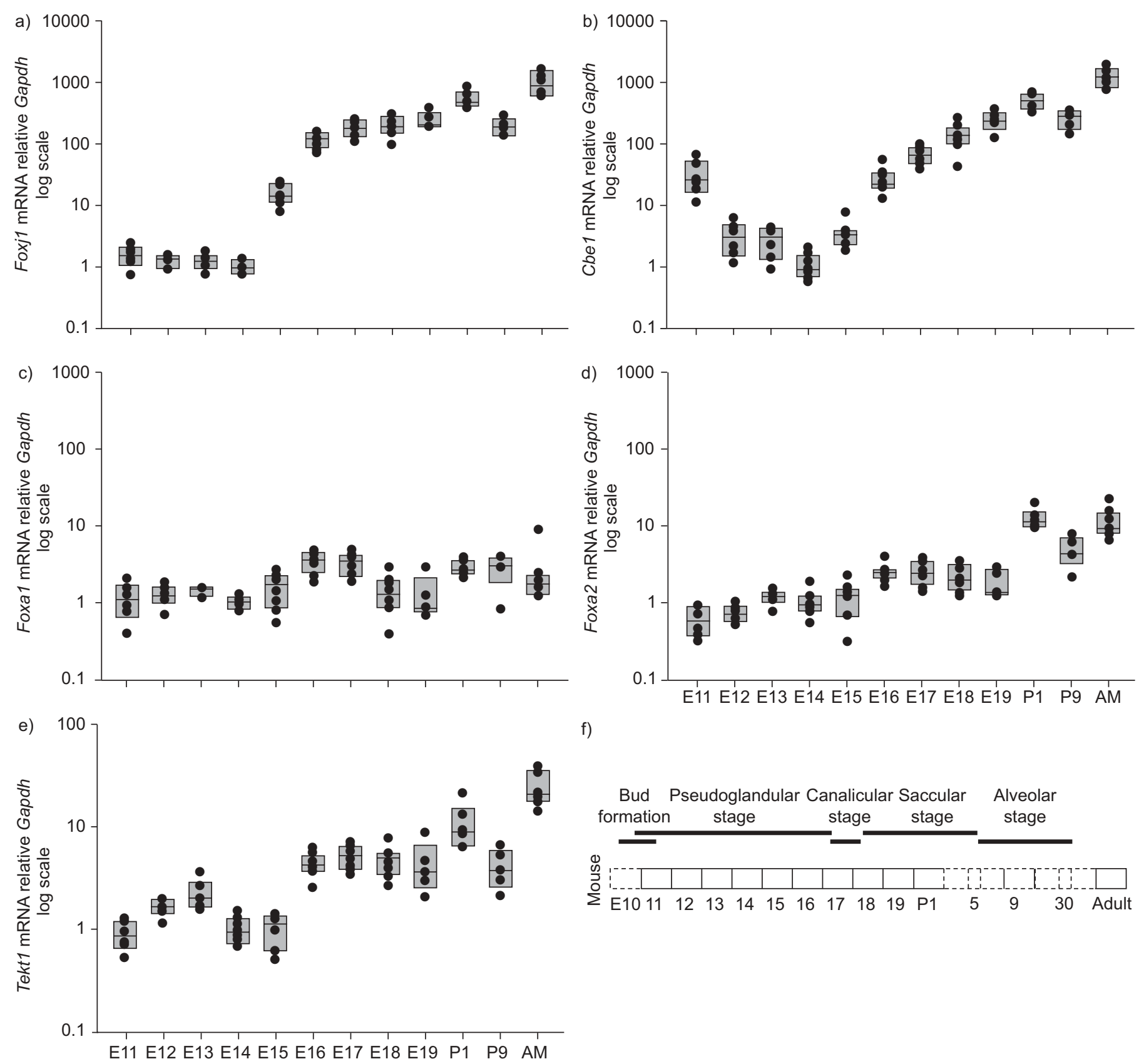

f)

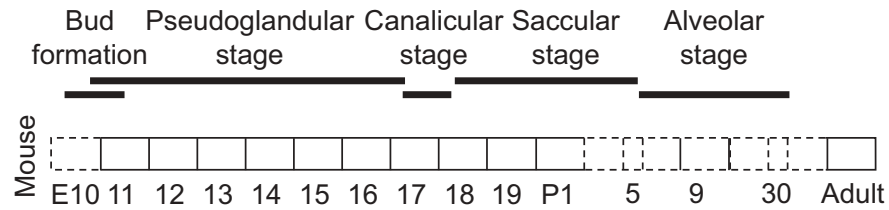

FIGURE 3. Chronological expression of a) Foxj1, b) Cbe1, c) Foxa1, d) Foxa2 and e) Tekt1 mRNAs during all stages of mouse lung development. f) Schematic representation of all stages of mouse lung development. Mouse lung tissues were dissected from embryos at the indicated days following gestation (embryonic day; E), from new-born mice at indicated days of post partum (P) and adult mice (AM). Total RNA was isolated and cDNA was synthesised for SYBR green-quantitative PCR analyses. The expression level is given relative to the level of Glyceraldehyde-3-phosphate dehydrogenase (Gapdh) mRNA, which was used as a housekeeping gene. Data are presented from five to eight lungs per group.

\section{Expression of CBE1 in embryonic/fetal lung tissue explant cultures}

Due to the difficulty in obtaining human fetal lungs after 1112 weeks post-conception, the current authors cultured embryonic/fetal lung tissues in vitro in order to mimic in vivo development and assessed the induction of CBE1 mRNA by RT-qPCR. Prior to culture, expression of CBE1 mRNA in embryonic lung obtained at 7-9 weeks post-conception was scarcely detectable (Ct values were $\sim 36-40$; data not shown), consistent with the semi-quantitative RT-PCR analyses (fig. 4a and b). However, when human fetal lungs at 9 weeks postconception were cultured in vitro, CBE1 mRNA was chronologically increased with a 10-fold increase in mRNA levels at day 12 (equivalent to 10.7 weeks post-conception in vivo; $\mathrm{p}=0.03$ ) and more than a 200-fold increase at day 18 (equivalent to 11.6 weeks post-conception in vivo; $\mathrm{p}=0.01$ ) compared to day 0 (fig. 6a). Expression of FOXJ1 also showed a parallel increase of $\sim 10$-fold at day $12(\mathrm{p}=0.01)$ and day 18 
a)

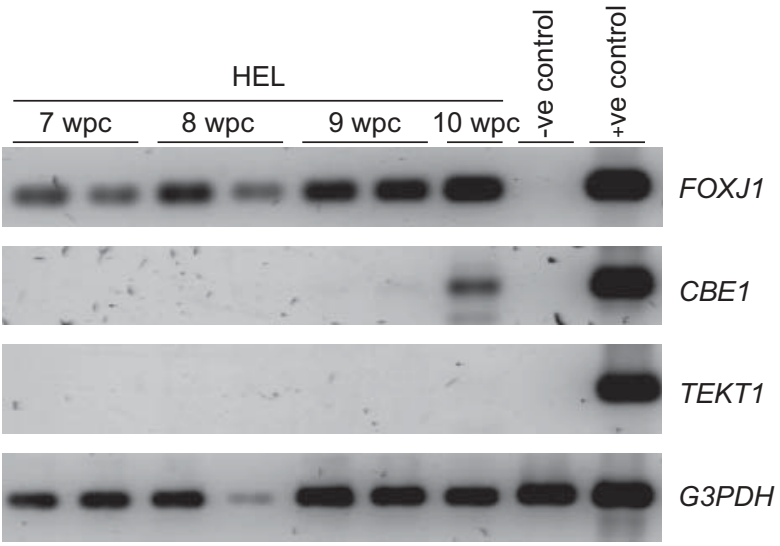

b)

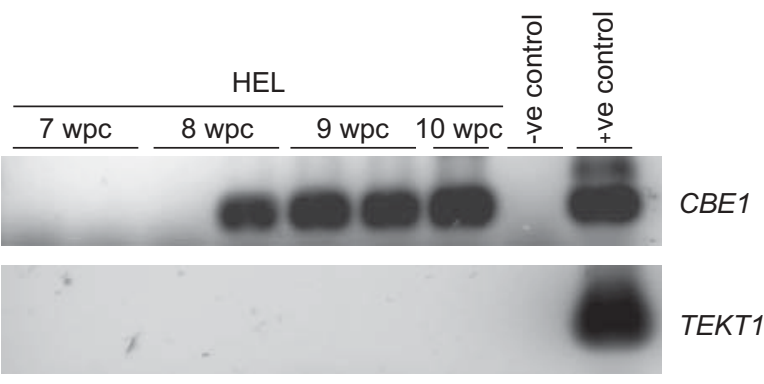

FIGURE 4. Expression of CBE1 mRNA in human embryonic/fetal lungs (HEL). a) Detection of mRNA analysed by semi-quantitative RT-PCR. Bronchial tissues were taken from HELs at 7, 8, 9 or 10 weeks post-conception (wpc). Each lane represents a different donor. PCR cycles were 35 for analysis of FOXJ1, CBE1 and TEKT1 and 25 for G3PDH cDNAs. CDNA from air-liquid interface-differentiated bronchial epithelial cells (at day 14) or cDNA from cultured bronchial fibroblasts was used as positive (+ve) or negative (-ve) control, respectively. b) Nested PCR (15 cycles) was carried out for CBE1 and TEKT1 mRNAs using diluted reaction products $(1: 10)$ of the standard RT-PCR analyses in (a) and the respective nested primers.

$(p=0.01)$, compared to day 0 (fig. $6 b$ ). The increase of expression levels was greater for CBE1 than FOXJ1 mRNA, probably because FOXJ1 was already significantly expressed before the start of culture at 9 weeks post-conception (fig. 4a). However, the present authors were unable to detect expression of TEKT1 mRNA during this ex vivo differentiation even after 18 days in culture (data not shown), confirming that expression of TEKT1 mRNA is absent when both CBE1 and FOXJ1 mRNAs are significantly expressed, as observed in the in vivo analyses (fig. $4 a$ and b). Protein expression of CBE1 in the cultured human fetal lungs was also investigated by immunohistochemistry, showing no staining at day 0 (9 weeks postconception) and day 6 (fig. $6 \mathrm{c}$ and d), but substantial staining in the developing epithelium at day 18 (equivalent to 11.6 weeks post-conception in vivo), when ciliary structures were visible (fig. 6e). Figure 7 shows a schematic representation summarising the temporal pattern of Cbe1/CBE1 expression in developing mouse and human lungs. Although the present authors were limited to obtaining human lung samples during the pseudoglandular stage of development, there was good concordance between the Cbe1/CBE1 expression profiles in both species.

\section{DISCUSSION}

In the healthy airways, ciliated cells represent $>80 \%$ of the total columnar epithelial cell population, and are interspersed with mucus-secreting goblet cells. However, in chronic airway diseases such as asthma, cystic fibrosis and chronic bronchitis, the number of goblet cells markedly increases [17, 18]; this results in accelerated production and/or secretion of mucus, which in turn causes resistance to air flow and abrogates normal mucociliary function. The consequences of these changes include increased sputum production, airway narrowing and disease exacerbation, or asphyxiation in the case of fatal asthma attacks [19]. Therefore, efficient and appropriate repair in bronchial epithelium, leading to enrichment of ciliated cells, would be of great benefit in airway diseases. However, the cellular and molecular mechanisms of ciliogenesis have not been fully investigated.

The factors required for the commitment of an undifferentiated airway epithelial cell to a ciliated cell are not fully known. As already indicated, studies using Foxj1 null mice, which fail to develop motile cilia, clearly show that Foxj1 plays a crucial role for ciliogenesis $[3,4]$. However, it is important to note that in Foxj1 $1^{-/-}$mice, cilia precursors are present inside airway epithelial cells but fail to dock at the apical membrane and form cilia [4]. Gain of function analyses using an adenovirus (or lentivirus) expression vector and ALI-cultured mouse tracheal epithelial cells has shown that Foxj1 alone is not sufficient to induce a program of ciliogenesis [5]. Indeed, in vivo and in vitro studies show that Foxj1 functions in the late stages of ciliogenesis to regulate basal body docking and axoneme formation in cells previously committed to the ciliated cell phenotype [20,21].

In order to study expression of Cbe1/CBE1 during lung development, the present authors used an in vivo approach with murine lungs and an ex vivo approach using human embryonic lung tissue explants. The explant culture provided a useful model for studying early human lung development. During the period of the experiments (up to 18 days), the present authors observed maintenance of branching morphogenesis. In terms of CBE1 protein expression, induction of expression after 18 days in vitro (9 weeks post-conception plus 2.6 weeks ex vivo) was similar to that observed in vivo at 12 weeks suggesting that this aspect of cellular programming was normal during the culture period. Although growth of the tissue eventually becomes limited by the requirement for a blood supply, this model offers the potential for studying molecular events that control the pseudoglandualr stage of development.

A previous study observed that transcription of CBE1, FOXJ1 and TEKT1 mRNAs was synchronous during in vitro differentiation using an ALI culture. All of these ciliated cellassociated genes were switched on at 14 days after the start of ALI, when RNA was extracted at day 7, 14 and 21 [6]. However, in murine lungs in vivo and using human fetal lungs ex vivo, a distinct order of expression was observed during differentiation of ciliated cells in the airway epithelium where Foxj1/FOXJ1 was earlier than Cbe1/CBE1 mRNAs, while expression of TEKT1 was undetectable $\leqslant 11-12$ weeks postconception. A further difference between the human adult and embryonic tissue was the protein distribution of CBE1. In adult 

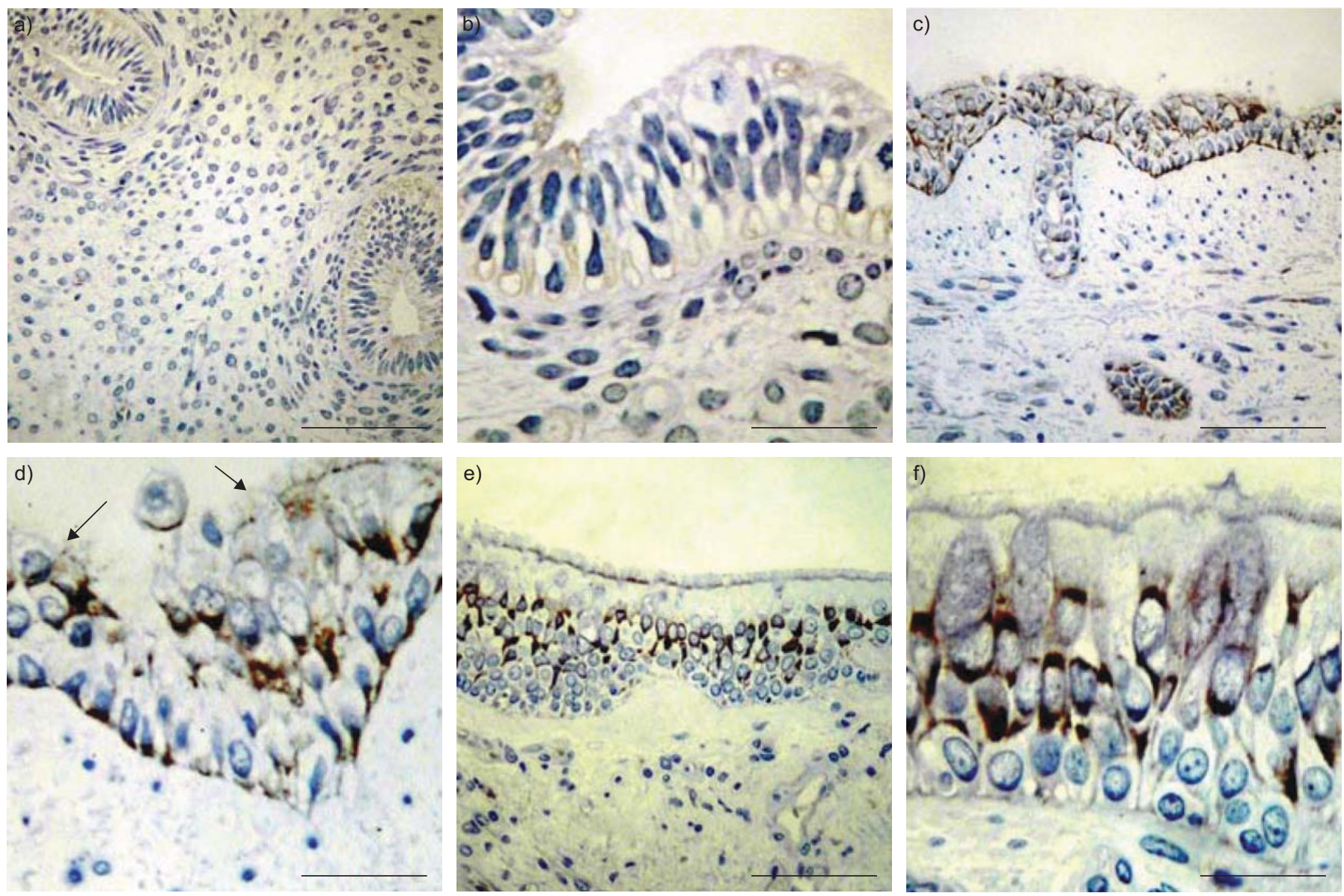

FIGURE 5. Expression of the CBE1 protein analysed by immunohistochemistry. Brown immunostaining using diaminobenzidine as chromagen shows the presence of CBE1 which is not detectable at 10 weeks post-conception ( $a$ and b), but is strong in epithelium at 12.3 weeks post-conception (c and d) where ciliary structures are visible (arrows). CBE1 immunostaining was detected not only in columnar cells but also basal cells. In contrast, staining is confined to columnar epithelial cells in adult human bronchi (e and f). a, c and e) Scale bars $=60 \mu \mathrm{m} ; \mathrm{b}, \mathrm{d}$ and f) scale bars $=20 \mu \mathrm{m}$.

bronchial epithelium, CBE1 protein immunostaining was restricted to the columnar epithelial cells, whereas in the embryonic lung tissue it could be detected in basal, as well as columnar, epithelial cells from 12 weeks post-conception. Whether these basal cells represent early ciliated cell progenitors within the pseudostratified epithelium remains to be determined. Their absence in adult bronchial epithelium may reflect slower cell turnover as compared with the much more rapidly growing embryonic airways. Alternatively, these findings may suggest that the in vitro differentiation system using adult cells does not necessarily reflect fetal lung differentiation, even though it produces fully differentiated columnar epithelial cells possessing beating cilia and mucussecreting goblet cells [22].

Table 1 shows a comparison of the stages of mouse and human lung development according to histological criteria [23-26]. Chronological expression of Foxj1 and Cbe1 mRNA in developing mouse lungs was consistent with that observed in human fetal airways, in that induction of Foxj1 was earlier (E15) than that of Cbe1 (E16) in late pseudoglandular stage of development (figs 3 and 7). The result obtained for Foxj1 expression is also consistent with a previous report showing that Foxj1 mRNA was detectable from E14.5 in embryonic lungs by Northern blot and in situ hybridisation analyses [27]. However, it should be noted that the present authors observed a biphasic expression of Cbe1 mRNA with significantly higher expression of Cbe1 at E11, during the formation of lung buds, when the expression of Foxj1 was absent (fig. 3ab \& 7). This suggests that $C b e 1$ may function during the early and later stages of lung development. E11 in mice corresponds to $4-5$ weeks post-conception in human embryos (table 1) and is a time when expression of FOXJ1 mRNA is absent [28]. Unfortunately, the present authors were unable to confirm CBE1 mRNA expression in human lungs as the appropriate embryonic tissues could not be obtained due to ethical reasons. Thus, whether human embryos at this early stage also transiently express CBE1 mRNA remains to be determined (fig. 7).

It was unexpected to find significant expression of Tekt 1 mRNA from E11 onwards because no TEKT1 mRNA was detected in human fetal lungs at 10 weeks post-conception, when both CBE1 and FOXJ1 mRNAs were observed. However, this chronological pattern of expression may be consistent with a previous study reporting, by Northern blotting, that Tekt1 mRNA was detectable from E12 onwards [29]. These data suggest that the regulatory mechanisms controlling transcription of TEKT1/Tekt1 mRNAs during embryogenesis 

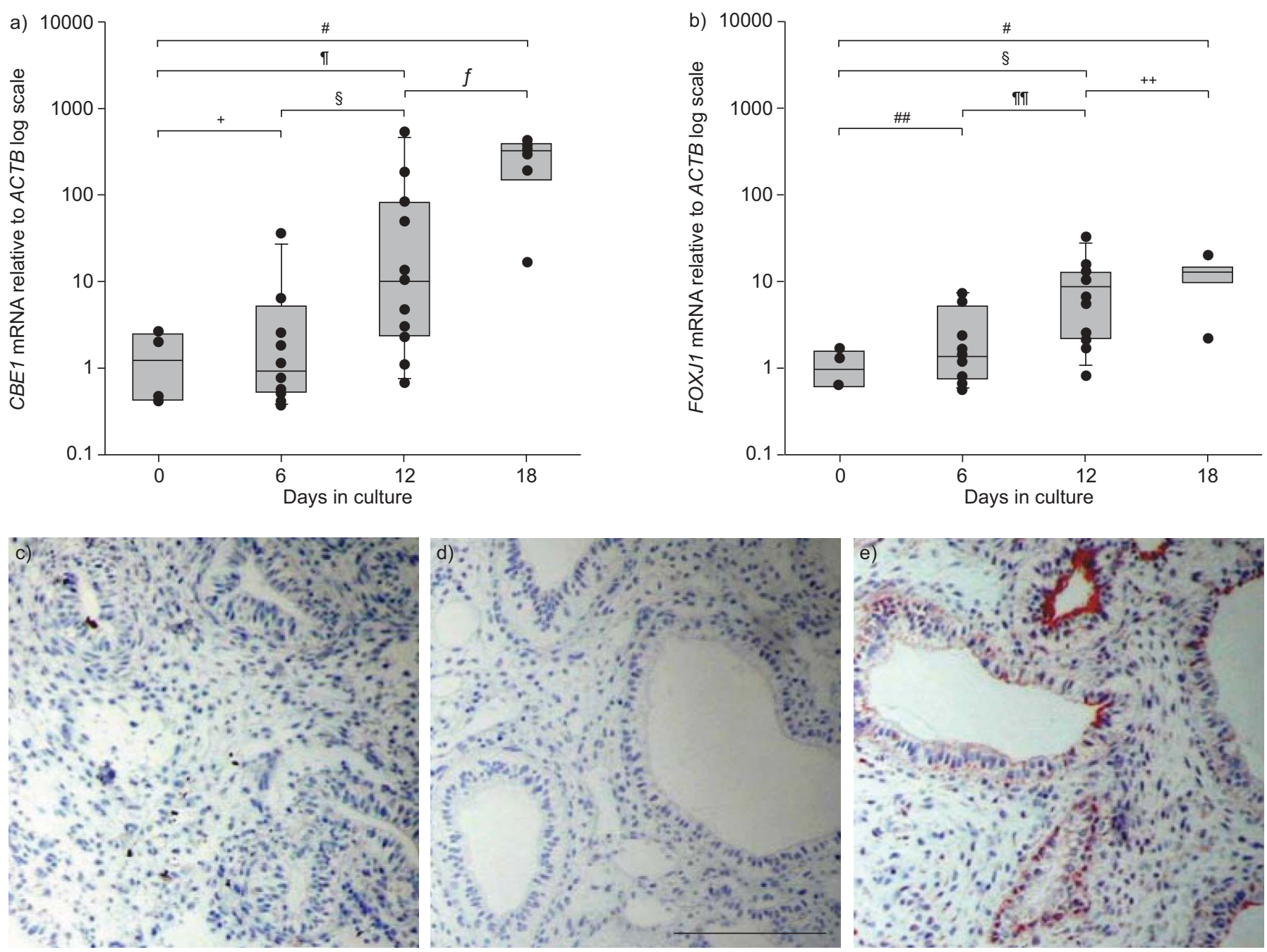

FIGURE 6. Chronological expression of a) CBE1 and b) FOXJ1 mRNAs during ex vivo differentiation of human embryonic/fetal lungs. mRNA expression was analysed by RT-quantitative PCR. Isolated human fetal lungs (7-9 weeks post-conception) were cultured and total RNA was isolated after the start of culture at the days indicated. Each data point represents the result obtained from an independent sample. Data are normalised relative to the housekeeping gene, $\beta$-actin (ACTB) mRNA levels. C-e) Protein expression was analysed by immunohistochemistry. Cultured (day 6 or $18 ; \mathrm{d}$ and e, respectively) or noncultured (day $0 ; \mathrm{c}$ ) embryonic lung tissues were fixed and stained as in figure 5; 3-amino-9-ethylcarbazole was used as chromagen to give positive red immunostaining. Scale bar=100 $\mu \mathrm{m}$.

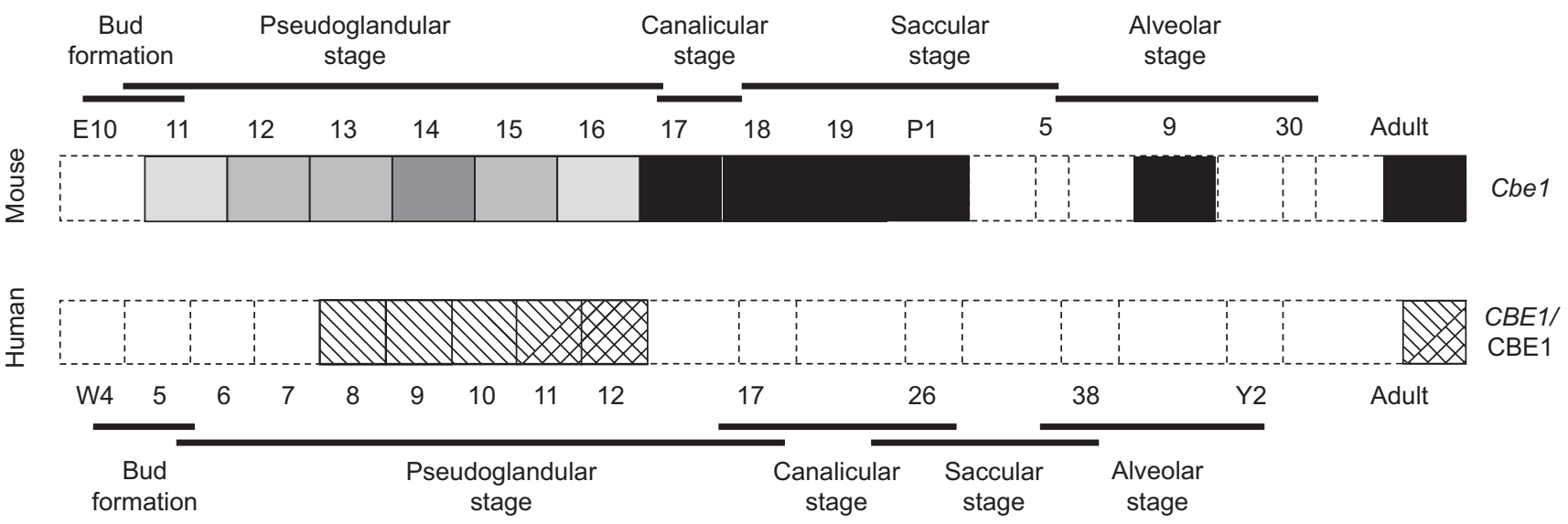

FIGURE 7. Schematic representation of stages of lung development and the temporal pattern of Cbe1 ( $\square, \square, \square$ and $\mathbf{\square})$ and CBE1 ( $\mathbb{Q}$ ) mRNA expression in mouse and

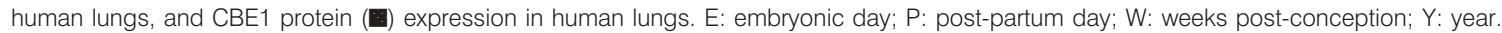


TABLE 1 Stages of mouse and human lung development post conception according to histological criteria

\begin{tabular}{lcc} 
Stage & Mouse & Human \\
\hline Bud formation start & $\sim 9.5$ days & 4 weeks $(\sim 26$ days $)$ \\
Pseudoglandular & $9.5-16.6$ days & $5-17$ weeks \\
Canalicular & $16.6-17.4$ days & $16-26$ weeks \\
Saccular & $17.4-5$ days after birth & $24-38$ weeks \\
Alveolar & Day 5-30 after birth & 36 weeks to $1-2$ yrs after birth \\
\hline
\end{tabular}

Data taken from [23-26].

are different between human and mice, although these orthologs are structurally highly conserved (82\% identical in amino acid sequences) [30].

Foxa1 and Foxa2, structurally homologous transcription factors, are now known to play a crucial role in the differentiation of bronchial epithelium. Conditional disruption of Foxa1 and Foxa2 reduced the expression of several marker genes in lung epithelium, including surfactant protein, Clara cell secretory protein and Foxj1 [12], suggesting that these transcription factors positively regulate expression of Foxj1 mRNA directly or indirectly. Protein expression of Foxa1 and Foxa2 in the mouse embryo has been precisely evaluated by immunohistochemistry, showing that both transcription factors can be detected in the nuclei in the lung bud at E10.5, and in the lung epithelium thereafter [13]. This is consistent with the present study which shows that Foxa1 and Foxa2 mRNAs were constantly detectable in the mouse embryonic lungs at E11 and thereafter (fig. $3 c$ and d). It has previously been reported that forced expression of FOXJ1 cDNA in a human bronchial epithelial cell line 16HBE 14o(-) induced endogenous TEKT1 mRNA expression but not $C B E 1$, indicating that FOXJ1 alone is not sufficient for the transcription of $C B E 1$ [6]. The presence of the Cbe1 mRNA at E11 may suggest that Cbe1 is induced by other transcription factor(s) such as Foxa1 or Foxa2 that function upstream of Foxj1. Chronological expression patterns during later stages of lung development may also suggest that Cbe1 cooperates with Foxj1 to control mucociliary differentiation. Recently, MATSUOKA et al. [31] described a spermatid specific gene, Smrp1, which is homologous to murine Cbe1. They reported three mRNA variants in the manchette, but only one of these transcripts was translated into protein. MATSUOKA et al. [31] also proposed that Smrp1 may be implicated in the formation of flagella or cilia constructed by tubulin.

Further functional studies are now required to define the role of Cbe1/CBE1 in lung epithelial differentiation and ciliogenesis.

\section{ACKNOWLEDGEMENTS}

The authors would like to thank R.M. Powell for designing and evaluating primers and probes for qPCR.

\section{REFERENCES}

1 Mills PR, Davies RJ, Devalia JL. Airway epithelial cells, cytokines, and pollutants. Am J Respir Crit Care Med 1999; 160: S38-S43.
2 Whitsett JA. Intrinsic and innate defenses in the lung: intersection of pathways regulating lung morphogenesis, host defense, and repair. J Clin Invest 2002; 109: 565-569.

3 Chen J, Knowles HJ, Hebert JL, Hackett BP. Mutation of the mouse hepatocyte nuclear factor/forkhead homologue 4 gene results in an absence of cilia and random left-right asymmetry. J Clin Invest 1998; 102: 1077-1082.

4 Brody SL, Yan XH, Wuerffel MK, Song SK, Shapiro SD. Ciliogenesis and left-right axis defects in forkhead factor HFH-4-null mice. Am J Respir Cell Mol Biol 2000; 23: 45-51.

5 You Y, Huang T, Richer EJ, et al. Role of f-box factor foxj1 in differentiation of ciliated airway epithelial cells. Am J Physiol Lung Cell Mol Physiol 2004; 286: L650-L657.

6 Yoshisue H, Puddicombe SM, Wilson SJ, et al. Characterization of ciliated bronchial epithelium 1, a ciliated cell-associated gene induced during mucociliary differentiation. Am J Respir Cell Mol Biol 2004; 31: 491-500.

7 Haitchi HM, Powell RM, Shaw TJ, et al. ADAM33 expression in asthmatic airways and human embryonic lungs. Am J Respir Crit Care Med 2005; 171: 958-965.

8 Britten KM, Howarth PH, Roche WR. Immunohistochemistry on resin sections: a comparison of resin embedding techniques for small mucosal biopsies. Biotech Histochem 1993; 68: 271-280.

9 Powell RM, Wicks J, Holloway JW, Holgate ST, Davies DE. The splicing and fate of ADAM33 transcripts in primary human airways fibroblasts. Am J Respir Cell Mol Biol 2004; 31: 13-21.

10 Kozak M. An analysis of 5'-noncoding sequences from 699 vertebrate messenger RNAs. Nucleic Acids Res 1987; 15: 8125-8148.

11 Wan $\mathrm{H}$, Kaestner KH, Ang SL, et al. Foxa2 regulates alveolarization and goblet cell hyperplasia. Development 2004; 131: 953-964.

12 Wan H, Dingle S, Xu Y, et al. Compensatory roles of Foxa1 and Foxa2 during lung morphogenesis. J Biol Chem 2005; 280: 13809-13816.

13 Besnard V, Wert SE, Hull WM, Whitsett JA. Immunohistochemical localization of Foxa1 and Foxa2 in mouse embryos and adult tissues. Gene Expr Patterns 2004; 5: 193-208.

14 Iguchi N, Tanaka H, Nakamura Y, Nozaki M, Fujiwara T, Nishimune Y. Cloning and characterization of the human tektin-t gene. Mol Hum Reprod 2002; 8: 525-530.

15 Gaillard DA, Lallement AV, Petit AF, Puchelle ES. In vivo ciliogenesis in human fetal tracheal epithelium. Am J Anat 1989; 185: 415-428. 
16 Jeffery PK. The development of large and small airways. Am J Resp Crit Care Med 1998; 157: S174-S180.

17 Rose MC, Nickola TJ, Voynow JA. Airway mucus obstruction: mucin glycoproteins, MUC gene regulation and goblet cell hyperplasia. Am J Respir Cell Mol Biol 2001; 25: 533-537.

18 Fahy JV. Goblet cell and mucin gene abnormalities in asthma. Chest 2002; 122: Suppl. 6, 320S-326S.

19 Aikawa T, Shimura S, Sasaki H, Ebina M, Takishima T. Marked goblet cell hyperplasia with mucus accumulation in the airways of patients who died of severe acute asthma attack. Chest 1992; 101: 916-921.

20 Huang T, You Y, Spoor MS, et al. Foxj1 is required for apical localization of ezrin in airway epithelial cells. J Cell Sci 2003; 116: 4935-4945.

21 Gomperts BN, Gong-Cooper X, Hackett BP. Foxj1 regulates basal body anchoring to the cytoskeleton of ciliated pulmonary epithelial cells. J Cell Sci 2004; 117: 1329-1337.

22 Puddicombe SM, Page A, Swallow DM, Thornton DJ, Holgate ST, Davies DE. Characterisation of the mucosecretory phenotype induced by chronic exposure to interleukin (IL)-13 in vitro. Am J Respir Crit Care Med 2003; 167: A454.

23 Strachan T, Lindsay S, Wilson DI, eds. Molecular Genetics of Early Human Development. Oxford, BIOS Scientific Publishers Ltd, 1997.

24 Rosenthal M, Bush A. The growing lung: normal development, and the long-term effects of pre- and postnatal insults. In: Bush A, Carlsen K-H, Zach MS. Growing Up with Lung Disease: the Lung in Transition to Adult Life. Eur Respir Mon 2002; 19: 1-24.

25 Perl AK, Whitsett JA. Molecular mechanisms controlling lung morphogenesis. Clin Genet 1999; 56: 14-27.

26 Ten Have-Opbroek AA. Lung development in the mouse embryo. Exp Lung Res 1991; 17: 111-130.

27 Hackett BP, Brody SL, Liang M, Zeitz ID, Bruns LA, Gitlin JD. Primary structure of hepatocyte nuclear factor/ forkhead homologue 4 and characterization of gene expression in the developing respiratory and reproductive epithelium. Proc Natl Acad Sci USA 1995; 92: 4249-4253.

28 Pelletier GJ, Brody SL, Liapis H, White RA, Hackett BP. A human forkhead/winged-helix transcription factor expressed in developing pulmonary and renal epithelium. Am J Physiol 1998; 274: L351-L359.

29 Norrander J, Larsson M, Stahl S, Hoog C, Linck R. Expression of ciliary tektins in brain and sensory development. J Neurosci 1998; 18: 8912-8918.

$30 \mathrm{Xu} \mathrm{M}$, Zhou Z, Cheng C, et al. Cloning and characterization of a novel human TEKTIN1 gene. Int J Biochem Cell Biol 2001; 33: 1172-1182.

31 Matsuoka Y, Miyagawa Y, Tokuhiro K, et al. Isolation and characterization of the spermatid-specific Smrp1 gene encoding a novel manchette protein. Mol Reprod Dev 2008; 75: 967-975. 